\title{
Mechanics of unloading of a rough surfaces pre-loaded joint
}

\author{
Peter Ogar ${ }^{1}$, Denis Gorokhov ${ }^{1, *}$, and Elena Ugryumova $^{1}$ \\ ${ }^{1}$ Bratsk State University, 665709 Makarenko 40, Bratsk, Russia
}

\begin{abstract}
In this paper we study the problem of the contact characteristics changing on a decrease of the load applied to the preloaded joint of roughness surfaces. The penetration of a rigid sphere (indenter) into the elastic hardenable half-space is considered originally. The elastic crater restoring by unloading is also considered. In elastic-plastic material's describing, Hollomon's pawer law is used. To describe a contact of a rigid rough surface with an elastic plastic half-space, the discrete model of a rough surface is used. Microasperities are represented as a set of identical spherical segments, the height distribution of which corresponds to the bearing profile curve of the surface. To describe the bearing profile curve, incomplete beta function ratio is used. The relations of relative contact areas $\eta$ and $\eta_{e}$ and gap densities at the joint $\Lambda_{e}$ and $\Lambda_{c e}$ on dimensionless loading $F_{q}$ and $F_{q e}$ at loading and unloading for different values of $\varepsilon_{y}$ and $\mathrm{n}$ are given. The obtained results are of practical importance for the sealing ability prediction of fixed sealing joints at the design stage, in particular for tightness supply of flange couplings and high pressure vessels seals.
\end{abstract}

\section{Introduction}

Many operational properties of joints of machine parts and devices, including tightness, are defined by contact interaction of rough surfaces [1]. In most cases, by contacting metal rough surfaces, the contact is elasticplastic. In $[2,3]$ for the description of an elastic-plastic contact, the authors use the kinetic sphere indentation's load-displacement diagram. As it follows from [1], the sealing ability of a sealing joint is defined by the relative contact area and the density of gaps in a joint. From this point of view, the loading of sealing joints at their assembly by the increased load and maintenance of their tightness by lower load to providing the minimum weight and size characteristics is of practical interest. Especially it is actual at the flange joints and high pressure vessels design.

To solve this problem it is necessary to know how the contact characteristics (the relative contact area and the density of gaps) change during unloading preloaded fixed joint.

\section{Contact of a rigid sphere with the elastic-plastic half-space}

Let us consider the indentation of the rigid sphere (indenter) into the elastic-plastic half-space and the elastic crater restoring. For the description of an elastic-plastic hardening material we use the Hollomon's power law

$$
S=\left\{\begin{array}{l}
\varepsilon E, \quad \varepsilon \leq \varepsilon_{y} \\
\sigma_{y}^{1-n} E^{n} \varepsilon^{n}, \quad \varepsilon>\varepsilon_{y},
\end{array}\right.
$$

where $n$ is the strain hardening exponent, $\varepsilon_{y}=\sigma_{y} / E$, $\sigma_{y}$ is the yield strength, $E$ is the elastic modulus.

By using a spherical indenter, the degree of deformation changes at all stage of loading and the primary result of such test is the kinetic indentation's loaddisplacement diagram [4] (Fig. 1).

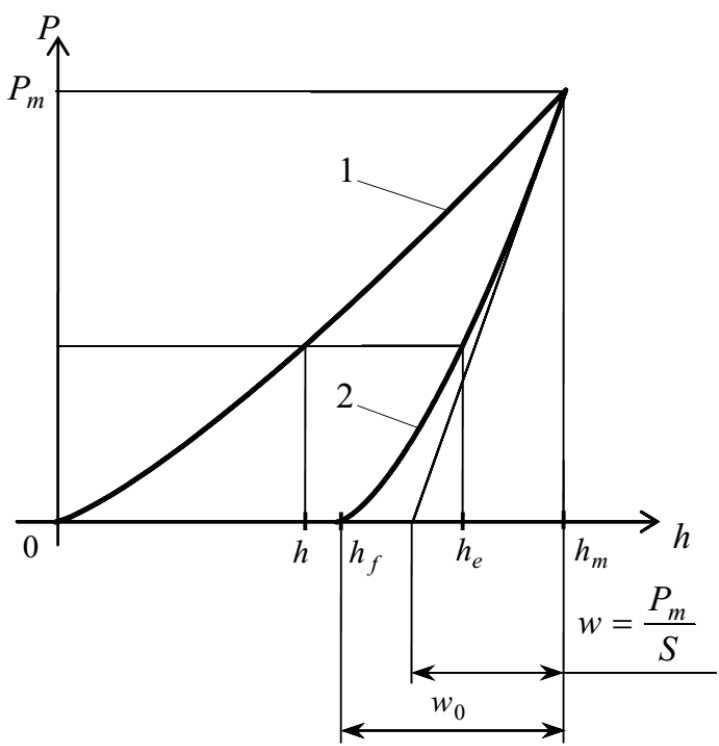

Fig. 1. The typical kinetic indentation's load-displacement diagram: 1 corresponds to loading, 2 corresponds to unloading

According to [3], the load-displacement branch can be written as

\footnotetext{
* Corresponding author: gorokhov@,brstu.ru
} 


$$
P=C_{1} h^{\alpha}
$$

and the unload-displacement branch can be written as

$$
P_{e}=P_{m}\left(\frac{h_{e}-h_{f}}{h_{m}-h_{f}}\right)^{\gamma}
$$

where $C_{1}, C_{2}$ are constants; $\alpha$ and $\gamma$ are exponents.

The initial unloading stiffness is

$$
S=\left.\frac{d P_{e}}{d h_{e}}\right|_{h_{c}=h_{s}}=\frac{P_{m} \cdot \gamma}{h_{m}-h_{f}}=\frac{P_{m} \cdot \gamma}{w_{0}} .
$$

From Fig. 1 follows that

$$
w=\frac{P_{m}}{S}=\frac{w_{0}}{\gamma}, \quad \gamma=\frac{w_{0}}{w} .
$$

In [3] parameter $\gamma$ is determined by means of a calculation

$$
\gamma=\frac{3-2 c^{2} \bar{h}_{m}}{2-c^{2} \bar{h}_{m}}
$$

where $\bar{h}_{m}=h_{m} / R, R$ is the sphere radius, $c^{2}=h_{c} / h, h_{c}$ is the depth at which the contact of an indenter and a material under load $P_{m}$ takes place.

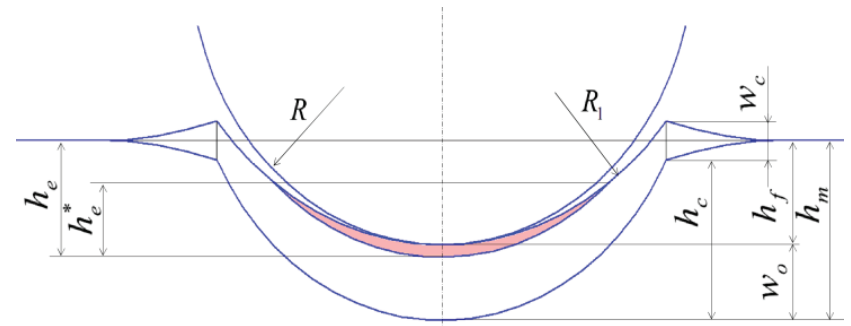

Fig. 2. Schematic illustration of a crater under unloading of a spherical indenter

The problem of determination of the contact area at elastic restoring was considered in [5], however the author did not take into account effects of "sink-in/pileup". Under loading to the contact depth $h_{e}^{*}$ (Fig. 2), the subsequent elastic restoring will be equal to $h_{e}-h_{f}$, in a similar under loading to the contact depth $h_{c}$, the elastic restoring will be equal to $h_{m}-h_{f}$. Let's make an assumption that the ratio takes place

$$
\frac{h_{e}^{*}}{h_{e}-h_{f}}=\frac{h_{c}}{h_{m}-h_{f}},
$$

then we have

$$
h_{e}^{*}=h_{c} \frac{h_{e}-h_{f}}{h_{m}-h_{f}}, \quad h_{f} \leq h_{e} \leq h_{m} .
$$

At $h_{e}=h_{f} \quad$ we $\quad$ have $h_{e}^{*}=0, \quad$ at $h_{e}=h_{m} \quad$ we have $h_{e}^{*}=h_{c}$, i.e. boundary conditions are carried out.

The contact area at crater restoring is

$$
A_{e}=\pi a_{e}^{2}=2 \pi R h_{e}^{*} .
$$

\section{Contact of a rigid rough surface with the elastic-plastic half-space}

\subsection{The discrete model of a rough surface}

Let us use the discrete model of a rough surface, in which microasperities are presented by identical spherical segments with the distribution of segments' peaks on height corresponds to the bearing profile curve of the real surface [1]. To describe the distribution of the reference curve is used incomplete beta function

$$
\eta(\varepsilon)=\frac{\mathrm{B}_{\varepsilon}(p, q)}{\mathrm{B}(p, q)}
$$

where $\mathrm{B}_{\varepsilon}(p, q)$ and $\mathrm{B}(p, q)$ are incomplete and complete beta functions;

$$
p=\left(\frac{R_{p}}{R_{q}}\right)^{2}\left(\frac{R_{\max }-R_{p}}{R_{\max }}\right)-\frac{R_{p}}{R_{\max }}, q=p\left(\frac{R_{\max }}{R_{p}}-1\right)
$$

where $R_{p}, R_{q}, R_{\max }$ are height roughness parameters according to ISO $4281 / 1-1997$.

In this case, the density of the asperities distribution on height function is

$$
\varphi_{n}^{\prime}(u)=\frac{u^{p-2}(1-u)^{q-2}[(p-1)(1-u)-(q-1) u]}{\varepsilon_{s}^{p-1}\left(1-\varepsilon_{s}\right)^{q-1}},
$$

where $\varepsilon_{s}$ is determined from the condition $\varphi_{n}\left(\varepsilon_{s}\right)=1$ [1]

Geometrical parameters of a spherical segment are: the height is $\omega R_{\max }$, where $\omega=1-\varepsilon_{s} ; a_{c}$ is the radius of the base of the spherical segment.

$$
R=\frac{a_{c}^{2}}{2 \omega R_{\max }}
$$

where $R>R_{\max }$.

At elastic contact, the relation between the relative size of indentation of the single asperity and the relative load is defined according to the Hertz theory.

$$
\frac{P_{e i}}{E^{*} R^{2}}=\frac{4}{3}\left(\frac{h_{i}}{R}\right)^{\frac{3}{2}}
$$

where $E^{*}$ is the reduced (contact) elastic modulus.

For elastic-plastic contact we use the equation from [6] 


$$
\frac{P_{e p i}}{E^{*} R^{2}}=e^{-B}\left(\frac{h_{i}}{R}\right)^{A},
$$

where $A=A\left(\varepsilon_{y}, n\right)$ and $B=B\left(\varepsilon_{y}, n\right)$.

\subsection{The relative contact area}

This problem has been considered in detail by [7]. When using the Eq. (14) for asperities of a rough surface, it is necessary to take into account that

$$
\begin{gathered}
h_{i}=(\varepsilon-u) R_{\max }, \\
\frac{h_{i}}{R}=\frac{(\varepsilon-u) \cdot 2 \omega R_{\max }^{2}}{a_{c}^{2}}=\left(\frac{\varepsilon-u}{2 \omega}\right) \cdot\left(\frac{2 \omega R_{\max }}{a_{c}}\right)^{2},
\end{gathered}
$$

where $\varepsilon$ is the relative approach a rough surface and the half-space; $u$ is the initial distance to the peak of $i$-th asperity.

When the indentation of a rigid rough surface is equal $\varepsilon$, the total load $P$ is described by equation

$$
P=\int_{\varepsilon-\varepsilon}^{\varepsilon} P_{e i} d n_{r}+\int_{0}^{\varepsilon-\varepsilon} P_{e p i} d n_{r},
$$

where $\varepsilon_{e}$ is the relative boundary an elastic contact, $d n_{r}$ is the number of peaks in a layer $d u$,

$$
d n_{r}=n_{c} \varphi_{n}^{\prime}(u) d u, n_{c}=\frac{A_{c}}{\pi a_{c}^{2}} .
$$

Let us consider a case, when $\varepsilon_{e} \rightarrow 0$.

Substituting Eq. (18) in Eq. (17), we have

$$
\frac{P}{A_{c}}=q_{c}=\int_{0}^{\varepsilon} q_{c e p i} \cdot \varphi_{n}^{\prime}(u) d u,
$$

where $q_{c e p i}=\frac{P_{e p i}}{\pi a_{c}^{2}}$.

By denoting

$$
\frac{q_{c} a_{c}}{\omega R_{\max } E^{*}}=F_{q}
$$

and by taking into account Eq. (14), (16), (19), we will finally obtain

$$
F_{q}(\varepsilon)=\frac{2^{2(A-1)} e^{-B}}{\pi}\left(\frac{\omega R_{\max }}{a_{c}}\right)^{2 A-3} \cdot \int_{0}^{\varepsilon}\left(\frac{\varepsilon-u}{2 \omega}\right)^{A} \varphi_{n}^{\prime}(u) d u .
$$

For the real contact area, similar to Eq. (17), we have

$$
A_{r}=\int_{0}^{\varepsilon} A_{r e p i} d n_{r} .
$$

Determining $A_{r i}$, we take into account that

$$
h_{c i}=c_{i}^{2} \cdot h_{i} .
$$

According to [8], we have

$$
c_{i}^{2}=\frac{h_{c i}}{h_{i}}=M^{\frac{2}{N}}\left(2 \frac{h_{i}}{R}\right)^{\frac{2}{N}-1},
$$

where $M=M\left(\varepsilon_{y}, n\right)$ and $N=N\left(\varepsilon_{y}, n\right)$.

For the relative contact area $\eta=A_{r} / A_{c}$, we will finally obtain

$$
\eta(\varepsilon)=(2 M) \frac{2}{N}\left(\frac{2 \omega R_{\max }}{a_{c}}\right)^{2\left(\frac{2}{N}-1\right)} \times \int_{0}^{\varepsilon}\left(\frac{\varepsilon-u}{2 \omega}\right)^{\frac{2}{N}} \varphi_{n}^{\prime}(u) d u .
$$

Let us consider the unloading process. For a single spherical asperity according to Eq. (3), we have

$$
P_{e i}=P_{e p i}\left(\frac{h_{e i}-h_{f i}}{h_{i}-h_{f i}}\right)^{\gamma} .
$$

For approximate calculations it is possible to accept $\gamma_{i} \approx 1,5$.

By taking into account that

$$
h_{i}-h_{f i}=w_{0 i},
$$

Eq. (3) can be written as

$$
P_{e i}=P_{e p i}\left(\frac{h_{i}-\Delta h-h_{f i}}{h_{i}-h_{f i}}\right)^{\gamma}=P_{e p i}\left(\frac{w_{0 i}-\Delta h}{w_{0 i}}\right)^{\gamma} .
$$

Similar to the equation Eq. (16) for $\Delta h / R$, we have

$$
\frac{\Delta h}{R}=\frac{\Delta_{\varepsilon}}{2 \omega}\left(\frac{2 \omega R_{\max }}{a_{c}}\right)^{2} .
$$

It should be noted that under unloading of a rigid rough surface, the size $\Delta h$ is identical for all asperities.

Using data from [2], we determine $w_{0 i}$ as

$$
\bar{w}_{0 i}=\frac{w_{0 i}}{R}=\frac{e^{B} \bar{h}^{A-\frac{1}{N}} K_{\alpha 0}}{\pi(2 M)^{\frac{1}{N}}},
$$

where $K_{\alpha 0}=2^{2 \alpha-1} \alpha \mathrm{B}(\alpha, \alpha), \alpha=A\left(\varepsilon_{y}, n\right)$.

By taking into account Eq. (16), we have

$$
\bar{w}_{0 i}=\frac{K_{\alpha 0} e^{-B}}{\pi(2 M)^{\frac{1}{N}}}\left(\frac{\varepsilon-u}{2 \omega}\right)^{A-\frac{1}{N}}\left(\frac{2 \omega R_{\max }}{a_{c}}\right)^{2\left(A-\frac{1}{N}\right)} .
$$

Then for unloading process, similar to Eq. (21), we will obtain 


$$
\begin{gathered}
F_{q e}\left(\varepsilon_{y}, n, \xi, \Delta_{\varepsilon}\right)=\frac{2^{2(\alpha-1)} e^{-B}}{\pi}\left(\frac{\omega R_{\max }}{a_{c}}\right)^{2 A-3} \times \\
\times \int_{0}^{\varepsilon}\left(\frac{\xi-u}{2 \omega}\right)\left(H\left(\varepsilon_{y}, n, \xi, u, \Delta_{\varepsilon}\right)\right)^{m} \varphi_{n}^{\prime}(u) d u,
\end{gathered}
$$

where

$$
H\left(\varepsilon_{y}, n, \xi, u, \Delta_{\varepsilon}\right)=\left\{\begin{array}{l}
\frac{w_{0 i}\left(\varepsilon_{y}, n, \xi, u\right)-\Delta_{\varepsilon}}{w_{0 i}\left(\varepsilon_{y}, n, \varepsilon, u\right)} \\
0, w_{0} \leq \Delta_{\varepsilon} . \\
w_{0}>\Delta_{\varepsilon}
\end{array}\right.
$$

Using Eq. (7) and Eq. (8), similar to Eq. (25), will have

$$
\begin{aligned}
& \eta_{e}\left(\varepsilon_{y},\right.\left.n, \xi, \Delta_{e}\right)=(2 M) \frac{2}{N}\left(\frac{2 \omega R_{\max }}{a}\right)^{2\left(\frac{2}{N}-1\right)} \times \\
& \times \int_{0}^{\varepsilon}\left(\frac{\xi-u}{2 \omega}\right)^{\frac{2}{N}} H\left(\varepsilon_{y}, n, \xi, u, \Delta_{\varepsilon}\right) \varphi_{n}^{\prime}(u) d u,
\end{aligned}
$$

where $H\left(\varepsilon_{y}, n, \xi, u, \Delta_{\varepsilon}\right)$ is defined by Eq. (33).

It should be noted that

$$
\left(\Delta_{\varepsilon}\right)_{\max }=\frac{w_{0 i}\left(\varepsilon_{y}, n, \xi, 0\right)}{2 \omega}\left(\frac{a_{c}}{R_{\max }}\right)^{2} .
$$

The fig. 3 shows the relations of loading $F_{q}(\varepsilon)$ and unloading $F_{q e}(\xi-\Delta \varepsilon)$ of a contact of a rigid rough surface with the elastic-plastic half-space for different values $\varepsilon_{y}$ and $n$ calculated on Eqs. (21) and (32).

The fig. 4 shows the relations the relative contact area $\eta$ and $\eta_{e}$ versus the dimensionless force elasticgeometric parameter $F_{q}$ and $F_{q e}$ under loading and unloading, respectively.

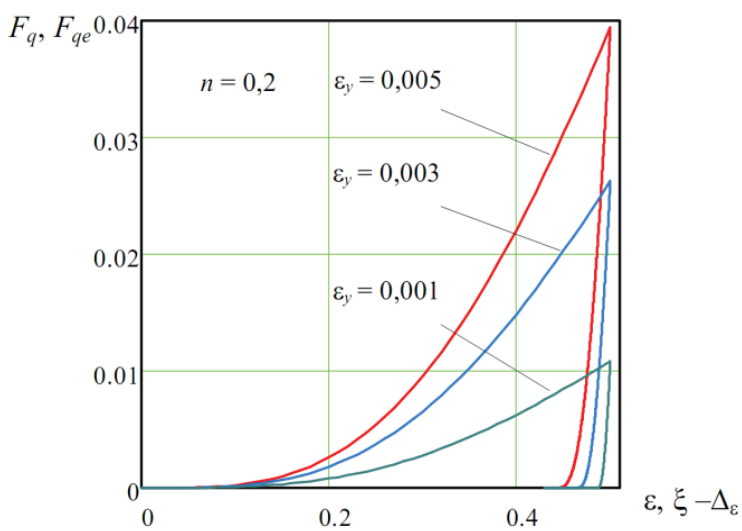

Fig. 3. The loading and unloading diagram of a contact of a rigid rough surface with the elastic-plastic half-space

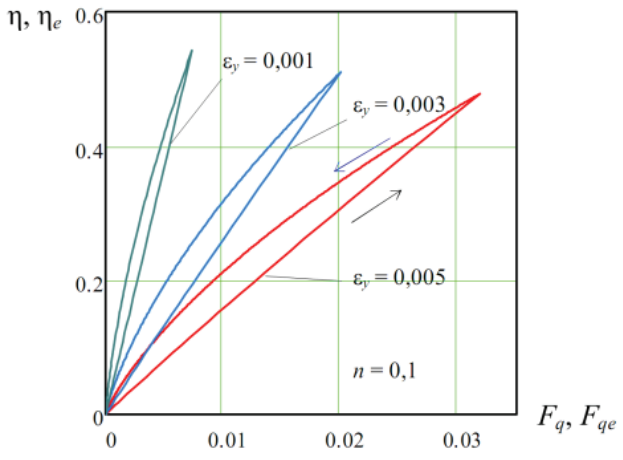

a

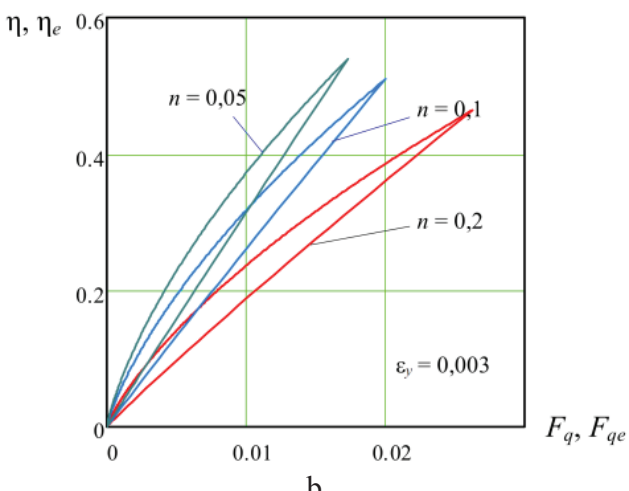

Fig. 4. The relative contact area versus the dimensionless force elastic-geometric parameter $\eta\left(F_{q}\right)$ and $\eta_{e}\left(F_{q e}\right)$ for different values $\varepsilon_{y}$ and $n$

\subsection{The density of gaps in the joint}

During indentation of rough surface on the value $\varepsilon R_{\max }$, contact of every single asperity is followed by "pileup/sink-in" effects, i.e. by plastic extrusion of the halfspace's material and its elastic squeezing [9]. For gaps volume is followed that

$$
V_{c}=A_{c} R_{\max }\left(1-K_{p}\right)-A_{c} \varepsilon R_{\max }+V_{e}-V_{p} .
$$

where $V_{e}$ is the total volume increase due to the elastic material squeezing for all the contacting asperities, $V_{e}$ is the total volume decrease due to the plastic material extrusion for all the contacting asperities.

Correspondingly, the joint's gaps density is

$$
\Lambda_{c}=\frac{V_{3}}{A_{c} R_{\max }}=1-K_{p}-\varepsilon+\Lambda_{e}-\Lambda_{p},
$$

where $\Lambda_{e}=V_{e} /\left(A_{c} R_{\max }\right), \Lambda_{p}=V_{p} /\left(A_{c} R_{\max }\right), K_{p}$ is the profile fill factor

$$
K_{p}=\int_{0}^{1} \eta(\varepsilon) d \varepsilon
$$

For a symmetric profile (when $\alpha=\beta$ ) $K_{p}=0,5$.

The total volume due to the elastic squeezing of all asperities is 


$$
V_{e}\left(\varepsilon_{y}, n, \varepsilon\right)=\int_{0}^{\varepsilon} V_{e i}\left(\varepsilon_{y}, n, \varepsilon, u\right) n_{c} \cdot \varphi^{\prime}(u) d u
$$

where

$$
V_{e i}=2 \pi \int_{a_{r}}^{a_{c}} r u_{z e}(r) d r
$$

$u_{z e}$ is the elastic displacement of surface points out of the contact area [10].

From the eq. for spherical segment volume with height $h_{f}$ and unloaded crater radius $\rho_{i}$, the volume of plastically displaced material, attributable to a single hole, is

$$
V_{p i}\left(\varepsilon_{y}, n, \varepsilon, u\right)=\pi \rho h_{f i}^{2}-\frac{\pi}{3} h_{f i}^{2}=\pi h_{f i}^{2}\left(\rho_{i}-\frac{h_{f i}}{3}\right)
$$

and the total displaced volume is

$$
V_{p}\left(\varepsilon_{y}, n, \varepsilon\right)=\int_{0}^{\varepsilon} V_{p i}\left(\varepsilon_{y}, n, \varepsilon, u\right) n_{c} \varphi_{n}^{\prime}(u) d u .
$$

Substituting Eq. (42) and Eq. (39) in Eq. (36), we determine the the density of gaps in the joint during rigid rough surface indentation into the elastoplastic hardening half-space as

$$
\Lambda_{c}\left(\varepsilon_{y}, n, \varepsilon\right)=1-K_{p}+\Lambda_{e e}\left(\varepsilon_{y}, n, \varepsilon\right)-\Lambda_{p}\left(\varepsilon_{y}, n, \varepsilon\right) .
$$

Carrying out similar arguments for the unloading process, we obtain

$$
V_{e e}\left(\varepsilon_{y}, n, \xi, \Delta_{\varepsilon}\right)=\int_{0}^{\xi} V_{e e i}\left(\varepsilon_{y}, n, \xi, u, \Delta_{\varepsilon}\right) n_{c} \cdot \varphi_{n}^{\prime}(u) d u
$$

The density of gaps in the joint during unloading is

$$
\begin{aligned}
& \Lambda_{c e}\left(\varepsilon_{y}, n, \xi, \Delta_{\varepsilon}\right)=1-K_{p}-\left(\xi-\Delta_{\varepsilon}\right)+ \\
& +\Lambda \\
& \left.\varepsilon_{y}, n, \xi, \Delta_{\varepsilon}\right)-\Lambda
\end{aligned}
$$

The fig. 5 shows the densities of gaps $\Lambda_{c}$ and $\Lambda_{c e}$ versus the dimensionless force elastic-geometric parameter $F_{q}$ and $F_{q e}$ under loading and unloading for different values $\varepsilon_{y}$ and $n$.

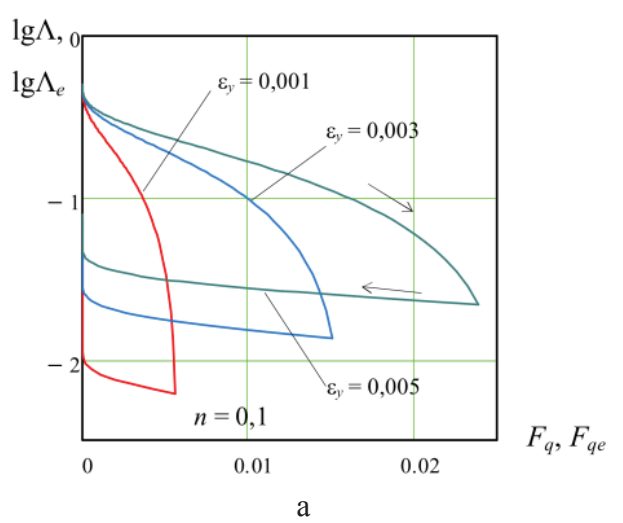

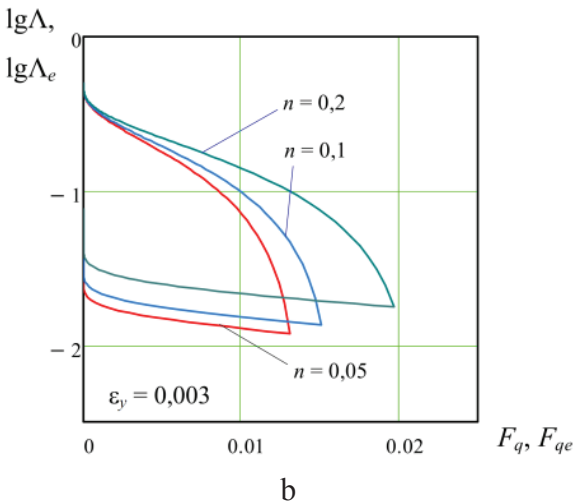

Fig. 5. The densities of gaps versus the dimensionless force elastic-geometric parameter $\Lambda_{c}\left(F_{q}\right)$ and $\Lambda_{c e}\left(F_{q e}\right)$ or different values $\varepsilon_{y}$ and $n$

\section{Application of the obtained results to practical calculations}

For example, the sealing ability of the joint of an elastoplastic contact is considered [11]. The intensity of the volume rate (per unit length along the seal perimeter) of ideally compressible gas through a sealing joint with a uniform distribution of contact pressure $q_{c}$ is

$$
Q=C_{u} \cdot C_{q}
$$

where $C_{u}$ is the dimensionless permeability functional that characterizes the sealing ability of the joint

$$
\begin{gathered}
C_{u}\left(\varepsilon_{y}, n, \varepsilon\right)=\frac{\left(\Lambda\left(\varepsilon_{y}, n, \varepsilon\right)\right)^{3} \cdot v_{n}\left(\varepsilon_{y}, n, \varepsilon\right)}{4\left(1-\eta\left(\varepsilon_{y}, n, \varepsilon\right)\right)^{2}}, \\
C_{q}=\frac{R_{\max }^{3}\left(p_{1}^{2}-p_{0}^{2}\right)}{2 l \mu},
\end{gathered}
$$

where $p_{1}$ is the environment pressure, $p$ is the atmosphere pressure, $\mu$ is the dynamic viscosity of the environment, $l$ is the width of the sealing area, $v_{n}$ is the rate of effective micro-channels.

At pressures above $16 \mathrm{MPa}$ at a leakage calculations, it should to use the model of a real gas [12].

The rate of effective micro-channels [11] is

$$
v_{n}\left(\varepsilon_{y}, n, \varepsilon\right)= \begin{cases}\left(1-x^{*}\right), & x^{*}<\frac{1}{3} \\ 3^{n-1}\left(1-x^{*}\right) n & x^{*} \geq \frac{1}{3}\end{cases}
$$

where $n$ is the number of rows of microasperities on the sealing zone width.

$$
x^{*}\left(\varepsilon_{y}, n, \varepsilon\right)=\int_{0}^{\varepsilon} x_{i}^{*}\left(\varepsilon_{y}, n, \varepsilon, u\right) \varphi^{\prime}(u) d u
$$

where 


$$
x_{i}^{*}\left(\varepsilon_{y}, n, \varepsilon, u\right)= \begin{cases}0, & \eta_{i}\left(\varepsilon_{y}, n, \varepsilon, u\right)<\eta_{i}^{*} \\ 1, & \eta_{i}\left(\varepsilon_{y}, n, \varepsilon, u\right) \geq \eta_{i}^{*}\end{cases}
$$

$\eta_{i}^{*}=0,5 \ldots 0,55$ is the critical value of $\eta_{i}$.

Using Eqs. (25), (43) and (49) - (51), it is possible to determine the dimensionless permeability functional during loading of the sealing joint. For unloading, it should to use Eqs. (36) and (45) and also to take into account that for determining of $v_{n e}\left(\varepsilon_{y}, n, \xi, \Delta_{\varepsilon}\right)$

$$
\eta_{e i}\left(\varepsilon_{y}, n, \xi, u, \Delta_{\varepsilon}\right)=\eta_{i}\left(\varepsilon_{y}, n, \xi, u\right) \cdot H\left(\varepsilon_{y}, n, \xi, u, \Delta_{\varepsilon}\right) .
$$

The fig. 6 shows the densities of gaps $C_{u}$ and $C_{u e}$ versus the dimensionless force elastic-geometric parameter $F_{q}$ and $F_{q e}$ under loading and unloading for different values $\varepsilon_{y}$ and $n$.
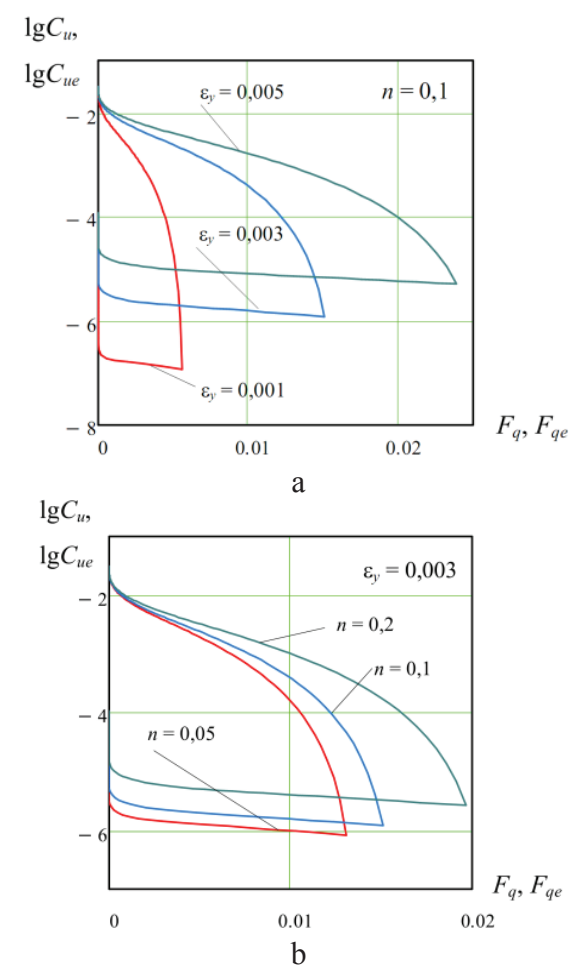

Fig. 6. dimensionless permeability functional versus the dimensionless force elastic-geometric parameter $C_{u}\left(F_{q}\right)$ and $C_{u e}\left(F_{q e}\right)$ for different values $\varepsilon_{y}$ and $n$

\section{Conclusions}

1. Using a discrete model of a rough surface, the authors obtained dependences for determining the relative contact area and the density of the gaps in the joint during indentation of a rigid rough surface into the elastic-plastic half-space and the subsequent unloading.

2. This makes it possible to predict the sealing ability of the sealing joint at various operating conditions, leading to unloading pre-loaded sealing joint.

3 . When load fully reduced, the functional permeability functional and hence the amount of leakage are reduced by about half order. This effect can be used to reduce the weight and size characteristics of the flanged joints and gaps of high-pressure vessels by loading it by an increased effort and maintaining required tightness by an lower effort.

4. The role of the material hardening parameters $\varepsilon_{y}$ and $n$ is shown. More efficient in terms of providing a predetermined tightness by an lower effort is $\varepsilon_{y}$.

\section{References}

1. N.B. Demkin, E.V. Ryzhov, Surface quality and contact of machine parts, 244 (Mashinostroyeniye, Moscow, 1981)

2. A.A. Lankov, Probability of elastic and plastic deformations while compressing metal rough surfaces, Treniye i smazka v mashinakh i mekhanizmakh 3, 35. (2009)

3. P.M. Ogar, V.A. Tarasov, Kinetic indentation application to determine contact characteristics of sphere and elastoplastic half-space, Adv. Mater. Res. 664, 625-631 (2013)

4. S.I. Bulychev, V.P. Alekhin,. Testing of materials by continuous depression of an indenter, 224 (Mashinostroyeniye, Moscow, 1990)

5. A.P. Kovalev, Basic laws of the indentation of a spherical indenter and evaluation of physical and mechanical properties of the surface layer of machine elements, Uprochnyayushchiye tekhnologii i pokrytiya 1, 36-41. (2007)

6. J.-M. Collin, G. Mauvoisin, P. Pilvin, Materials characterization by instrumented indentation using two different approaches, Materials and Desing, 31, 636640 (2010)

7. P.M. Ogar, D.B. Gorokhov. Influence of materials hardenability parameters on the machine parts characteristics after unloading, Key Engineering Materials 723, 369-375 (2017)

8. X. Hernot, O. Bartier, Y. Bekouche, R. El Abdi, G. Mauvoisin, Influence of penetration depth and mechanical properties on contact radius determination for spherical indentation, Int. Journal of Solids and Structures, 43, 4136-4153 (2006)

9. P.M. Ogar, V.A. Tarasov, A.V. Turchenko, Contact of a rigid rough surface with elastic-plastic half space, Systems. Methods. Technologies, 13, 17-22 (2012)

10. P.M. Ogar, V.A. Tarasov, Influence of axisymmetric form of load on stressedly-deformed state of elastoplastic half-space, Systems. Methods. Technologies 5, 14-20 (2010)

11. Yu.N. Alpatov, V.A. Tarasov, A.V. Turchenko, Impact of the hardenable material characteristics on the joints sealing property, Sovremennye tekhnologii. Sistemny analiz. Modelirovaniye, 2, 8388 (2012)

12. D.B. Gorokhov, A.A. Sobolevsky, Leakage variation of gas environment through the joint of releasable connections with temperature increasing, Sovremennye tekhnologii. Sistemny analiz. Modelirovaniye, 3, 70-76 (2016) 